\title{
1 Evaluating Enzymatic Synthesis of Small Molecule Drugs
}

3 Matthew Moura*, Justin Finkle ${ }^{\dagger}$, Sarah Stainbrook $^{\dagger}$, Jennifer Greene*, Linda J Broadbelt ${ }^{* \dagger}$, 4 Keith EJ Tyo ${ }^{*+\dagger}$

5

6

7 8
* Department of Chemical and Biological Engineering, Northwestern University, Evanston, IL, 60208

$\dagger$ Interdisciplinary Biological Sciences Graduate Program, Northwestern University, Evanston, IL, 60208
† Corresponding authors

2145 Sheridan Road

Technological Institute

Evanston, IL

60208

KEJT: (847) 868-0319

LJB: (847) 491-5351 


\section{$44 \quad \underline{\text { Abstract }}$}

There have been many achievements in applying biochemical synthetic routes to the 46 synthesis of commodity chemicals. However, most of these endeavors have focused on 47 optimizing and increasing the yields of naturally existing pathways. We sought to evaluate the potential for biosynthesis beyond the limits of known biochemistry towards the production of 49 small molecule drugs that do not exist in nature. Because of the potential for improved yields compared to total synthesis, and therefore lower manufacturing costs, we focused on drugs for

51 diseases endemic to many resource-poor regions, like tuberculosis and HIV. Using generalized

52 biochemical reaction rules, we were able to design biochemical pathways for the production of 53 eight small molecule drugs or drug precursors and identify potential enzyme-substrate pairs for 54 nearly every predicted reaction. All pathways begin from native metabolites, abrogating the need 55 for specialized precursors. The simulated pathways showed several trends with the sequential 56 ordering of reactions as well as the types of chemistries used. For some compounds, the main 57 obstacles to finding feasible biochemical pathways were the lack of appropriate, natural starting 58 compounds and a low diversity of biochemical coupling reactions necessary to synthesize 59 molecules with larger molecular size.

60

61

62

63

64

65 Keywords: Metabolic engineering, computational biology, predictive biochemistry, 66 pharmaceutical production, drug synthesis 

74 others. $^{6}$

\section{$\underline{\text { 1. Introduction }}$}

While organic synthesis is the preferred route for production of small molecule drugs, engineered biosynthesis schemes have shown great promise in changing how drugs can be made. Paclitaxel $^{1}$ and $\operatorname{artemisinin}^{2}$ are two examples in which biochemical pathways were heterologously expressed to dramatically increase available yields. In addition to these two endeavors, enzymes have shown great promise as biocatalysts for single-step reactions in drug synthesis schemes for selective reductions ${ }^{3}$, transaminations ${ }^{4}$, enantioselective purifications ${ }^{5}$, and

Enzyme-driven catalytic schemes for small molecules benefit from many advantages. Enzymes natively operate in aqueous conditions, ambient temperatures, and at moderate $\mathrm{pH}$, which can simplify manufacturing and reduce waste treatment requirements. Additionally, the specificity and selectivity of enzymes are typically higher than that achieved through traditional chemical synthesis routes. Enzymes thus have the potential to reduce byproducts, especially in the case of in vitro reaction systems. Thus far, however, biosynthetic applications to drug synthesis have focused on either using enzymes for single steps, as previously mentioned, or for the production of natural product drugs. To date, there has been no evaluation of biosynthesis for the full synthesis of non-natural small molecule drugs.

As non-natural biosynthesis pathways will inherently depend on enzymatic activity against non-native substrates, the concept of enzyme promiscuity will be vital for successful novel pathway development. Enzyme promiscuity can be broadly classified as either catalytic or substrate promiscuity ${ }^{7}$, where an enzyme either catalyzes a distinct chemistry from that which it typically performs (catalytic) or catalyzes the same basic chemical reaction but on a variety of substrates (substrate). Studies have shown that promiscuity is much more permissive than has 
90 been previously thought. ${ }^{8}$ As advances in genomic sequencing and enzyme discovery progress,

91 the coverage of permissible enzymatic chemistry will only grow; however, so too will the

92 difficulty in abstracting this information. Computational tools will therefore be necessary to

93 successfully find enzyme candidates capable of performing these promiscuous reactions.

Predictive biochemistry approaches are required for the in silico design of these types of

95 pathways. There are many tools for the computational design of biochemical pathways, as have

96 been reviewed by our group ${ }^{9}$ and others, ${ }^{10,11}$ but the majority of these techniques optimize

97 preexisting metabolic networks for maximizing production levels of known metabolites. Greater

98 use of the tools available for metabolic engineering is necessary to continue the growth of this

99 field towards the novel bioproduction of chemicals. ${ }^{12}$ Some examples of techniques that predict

100 biochemical reactions include XTMS, ${ }^{13} \mathrm{MINE}^{14}$ the KEGG PathPred system ${ }^{15}$, Gem-Path ${ }^{16}$, and

101 the tools utilized in this paper - the Biochemical Network Integrated Computational Explorer

102 (BNICE) $^{17}$ and SimZyme ${ }^{18}$. In these cases, biochemistry is distilled into generalized reaction

103 rules which are applied to putative substrates to predict potential enzymatic chemistry. The

104 BNICE software package has been well-documented as a predictor of experimentally validated

105 biochemistry. ${ }^{18-21}$

106

In this work, biosynthetic routes for non-natural drug molecules with confirmed clinical

107 relevance were developed. Using BNICE, reaction networks that define the biochemical 108 reactions of each step were designed for several drug molecules. SimZyme ${ }^{18}$, which identifies 109 specific isoenzymes for a particular biochemistry, was then used to identify enzyme candidates 110 for nearly every reaction step. The general process is summarized in Figure 1. To ensure that 111 pathways were completely biosynthetic, successful networks all begin from molecules either 
112 known to exist within the metabolome, or from molecules with previously demonstrated 113 heterologous production routes.

114 2. Methods

$115 \quad 2.1$ Assembling an Initial List of Candidate Molecules

To develop a list of candidate small molecules that would benefit from reduced 117 manufacturing costs that may be afforded by biochemical synthesis, multiple sources were 118 utilized. First, the World Health Organization's (WHO) Global Price Reporting Mechanism ${ }^{22}$ 119 was used to identify drugs that consumed large portions of the WHO's expenditures in resource 120 poor regions. To identify targets with costs infeasible for mass distribution systems, experts 121 within the Northwestern Center for Global Health, the Bill and Melinda Gates Foundation, the 122 Clinton Global Initiative, and the TB Alliance were also consulted. From this search, a total of 12313 molecules were chosen as simulation targets (Supplementary Table A). ${ }^{17}$ All targets were 124 selected based on preexistent pharmaceutical relevance, and on having no known methods for 125 synthesizing the chemicals through fully biosynthetic means.

\subsection{The BNICE Framework}

Reaction networks were generated with BNICE as has been described previously ${ }^{9,17,19,23}$. In brief, the system uses a set of hand-curated reaction rules, termed "operators", based on the 129 Enzyme Commission (EC) nomenclature for enzymatic reactions. Operators recognize molecular substructures with demonstrated activity within defined EC classes and search for these patterns 131 on putative substrates. These substructures can include reacting as well as spectator atoms. The operator performs the biochemical reaction common to that EC class on the defined substructure, 133 including the use of any required cofactor molecules, like ATP or NADH, as dictated by the EC 134 entries used to originally define the operator. Using all operators available within the lab from 
135 previous research ${ }^{19-21}$, these reaction rules were applied iteratively to starting compounds in 136 either forward or reverse (retrosynthetic) directions. Additionally, SimIndex ${ }^{18}$ was used to allow 137 for more efficient and extensive network exploration by pruning networks based on the chemical 138 similarity of pathway intermediates to the desired product.

139

\subsection{Identifying Starting Molecules from Native Metabolism}

It was desirable for networks and pathways to connect directly to native metabolites. This would provide a template for future experimental works, where drug molecules could be synthesized without the need for potentially expensive chemical additives to cell culture, or the need for transporting additives into a cellular environment.

Two methods were used to identify biological starting substrates for the drug pathway simulations. Target drug products were analyzed with Kyoto Encyclopedia of Genes and Genomes (KEGG) SimComp ${ }^{24}$ to identify chemically similar naturally occurring metabolites. Alternatively, a retrosynthetic BNICE simulation, with the operators altered to encode the reverse reaction from the originally stated direction, was run to identify hypothetical precursors to the target compound. Compounds predicted in the retrosynthetic network were compared against metabolites found in the iAF1260 Escherichia coli metabolic model ${ }^{25}$ or the full KEGG database. $^{26}$

\subsection{Identifying Biochemical Pathways and Networks between Starting Compounds and} Drug Product

If a candidate metabolite was found with SimComp, then a metabolic reaction network was generated using BNICE and biased by SimIndex ${ }^{18}$ to save computational effort. If this search did not succeed in resolving to the desired product, a retrosynthetic simulation on the product was run. The forward search network from the SimComp substrate and the retrosynthetic 
172 feasibility. ${ }^{23}$

177 Alafenamide (TAF) and Tenofovir Disoproxil (TDF). after the network generation had completed. ${ }^{18,27}$

network from the desired drug product were then matched to search for overlapped chemical intermediates and merged into a single network. Pathways from the candidate metabolite to the final drug product were searched for within this merged network. The pathway search used a Byers-Waterman algorithm to find optimal and near-optimal pathway solutions with pathway lengths at or near the generational step where the product was generated, and was performed

In the event multiple pathways were found, we used several criteria to prioritize the pathways most amenable to experimental implementation. Pathways with fewer enzymatic steps were prioritized to mitigate uncertainty associated with promiscuous predictions, and inefficiencies that may be associated with long pathways (in terms of increased demand for enzyme synthesis and reduced product yield due to intermediates lost to diffusion). The shortest successful pathways of those that were found are presented in this paper, though some longer examples of note are also included in the supplement. Group contribution thermodynamic calculations were also performed on all reactions to predict the $\Delta G_{r}$ for each step to evaluate

For the particular case of tenofovir, successful pathway development required the use of an expanded operator set. During the course of this work, a more broadly-defined operator set, developed elsewhere ${ }^{28}$ was successfully used to predict the synthesis of tenofovir. Additionally, this set was also used to develop pathways for two prodrug forms of this molecule: Tenofovir

\subsection{Identification of Enzyme Candidates for Reactions}

Using the predicted substrates and EC class from BNICE, enzymes likely to carry out the desired chemistry on the substrate were identified using SimZyme ${ }^{18}$ and the BRENDA ${ }^{29}$ 
181 database. FP2 and FP4-based fingerprints were both used to calculate Tanimoto coefficients ${ }^{30,31}$ 182 between the predicted and known substrates from BNICE and BRENDA, respectively. The 183 SimZyme output thus gives a ranked list of all substrates within all BRENDA entries that share 184 the 3-digit EC values as dictated by BNICE. For each substrate in the SimZyme list, the specific 185 enzyme (4-digit EC) entry is indicated, which can then be used to find the particular isozyme 186 candidates that may be active against the specific substrate. Two chemical transformations did 187 not use this strategy, both within the tert-leucine biosynthesis: (a) the keto-enol shift of the 188 substrate for step 16 was assumed to occur spontaneously, and (b) the decarboxylation class 189 identified for step $\mathbf{1 7}$ typically requires an aromatic ring, which was not the case for the putative 190 substrate. However, evidence exists for chemical and biological means for the successful 191 decarboxylation of $\alpha$-ketoacids, though it may involve an aldehyde intermediate, which would be 192 easily oxidized to the desired carboxylic acid. ${ }^{32,33}$

193 Tables 1-3 contain the identified 4-digit EC classes for the three presented pathways in 194 this work, as well as the paired BNICE and BRENDA substrates. Supplementary B contains 195 this same information, the thermodynamic values, and the parent organisms of the EC numbers 196 for the BRENDA-matched substrates.

$197 \quad 2.6$ Yield Calculations

198 Yield analysis was conducted in a similar manner to previous publications. ${ }^{19}$ Each overall 199 BNICE-generated design pathway was condensed into a single pathway. including the pathway's 200 starting compounds and the drug molecule of interest as the product. The total number of 201 cofactor-pairs (e.g. ATP/ADP, NADH/NAD) consumed and produced across each overall 202 pathway were also included, as well as any other products released by the pathways. 
To calculate the max theoretical yield of each drug molecule, a flux balance analysis

204

205

206

207

208

209

210

211

212

213

214

215

216

217

218

219

220

221

222

223

224

225

calculation was completed for each pathway using the COBRA toolbox for MATLAB ${ }^{34}$. For each drug, its respective condensed pathway is inserted into the iAF $1260^{25}$ genome-scale metabolic model of E. coli. Although all starting substrates have been documented to occur in metabolism, not all are within the iAF1260 model. For those cases, either import reactions were added to allow for the "input" of the precursor, or additional reactions were added to connect the starting molecule to metabolites present in iAF1260. These cases are noted in Table 4, along with the reported maximum theoretical yield values. For each yield calculation, either the uptake flux of either glucose or the import substrate is set to $1 \mathrm{mmol} / \mathrm{gDCW} / \mathrm{hr}$. For all pathways, the objective function for the model was set to maximize flux through the condensed drug pathway.

\section{$\underline{\text { 3. Results }}$}

Of the list of 13 tested molecules, successful pathways were developed for 8 compounds entirely composed of predictive biochemical reactions. These pathways all stem from metabolites known to exist biochemically and have been assigned individual enzyme classes. Each predicted reaction was linked to enzyme-substrate pairs with demonstrated activity on BRENDA, as well as chemical substrate similarity. Several of these pathways are presented below, while the rest can be found in Supplementary F and G.

\subsection{Pyrazinamide}

For pyrazinamide, a member of the tetrad combination treatment for M. tuberculosis, pyrazine, while not a known natural metabolite, was selected as the starting substrate. Initial pathway analysis identified methyl pyrazine as an intermediate. Because methyl pyrazine is known to be produced by Corynebacterium glutamicum, ${ }^{35}$ in practice methyl pyrazine may be preferable to unsubstituted pyrazine as a starting compound. 
BNICE simulations gave a range of pathways, with the shortest pathways we were able to

227 find shown in Figure 2. Although the pathways for pyrazinamide showed significant branching

228 in the larger network (Supplementary Figure E), all but one of these pathways converged to

229 that presented in Figure 2. This starts with the methylation of the pyrazine ring in step 1,

230 followed by a number of oxidation steps to yield a carboxylic acid that is aminated to give the

231 final drug product. Two short pathway branches are also shown in Figure 2. A single divergent

232 pathway was seen in the expanded network in Supplementary E, where steps $\mathbf{4}$ and $\mathbf{5}$ were

233 replaced with a six-step pathway bypassing pyrazinoic acid. Matched substrates, mostly 234 consisting of varying substituted aromatic ring structures, can be seen in Table 1.

\subsection{L-Tert Leucine}

Searching for pathways for the synthesis of L-tert leucine was motivated by its use as a

237 key precursor in the chemical synthesis of atazanavir, an HIV protease inhibitor for which we 238 could not find a biochemical synthesis pathway. L-tert leucine is a non-biological amino acid 239 with an isobutyl sidechain. This molecule has also demonstrated utility in the synthesis of cancer 240 chemotherapeutics $^{36}$, additional antivirals ${ }^{6,37}$, and for asymmetric organocatalysis. ${ }^{5}$ Using a 241 SimComp search for tert-leucine, 2-dehydropantoate was selected as a starting substrate due to it 242 having a quaternary carbon center. By combining forward and retrosynthetic simulations, paths 243 were found in as few as eight steps.

245 reactions is fairly low. Pathways from 2-dehydropantoate to tert-leucine consist of either seven 246 or eight biochemical reactions (not including step $\mathbf{1 6}$ which is a spontaneous keto-enol 247 tautomerism). In the first half of the network, steps 1-11, all of the reactions are one of three 248 chemical transformations - an oxidoreduction (e.g., step 1), an aminotransferase (e.g., step 2), or 
an aldehyde C-C acetyl transferase (e.g., step 4). These three chemistries occur largely independent of order (though an aldehyde is a prerequisite for the acetylation).

For the remaining reaction steps, the previous parts of the pathways converged to a 252 stricter set of ordered steps. With the lengthened carbon chain, the previously primary, now 253 secondary, alcohol on the tert-butyl group is "shifted" farther along the chain, through an 254 additional hydroxylation in steps 12-15, though even here the order of hydroxylation and 255 dehydration is flexible. This pseudo-isomerization is necessary because the adjacent quaternary 256 carbon prohibits direct dehydration to a double bond. The new structure is then decarboxylated 257 twice to yield the final drug product. All predicted substrates were successfully matched to 258 BRENDA substrates (Table 2), although the match for step $\mathbf{1 7}$ was in a class of enzymes that 259 had only demonstrated activity for aromatic substrates. Though this does not preclude the 260 possibility for an enzyme to perform this reaction, further work is necessary to determine the 261 necessity of an aromatic backbone for this reaction.

In addition to the dehydropantoate pathway shown in Figure 3, an analysis of the 263 retrosynthetic network alone suggested an alternative synthesis scheme. The retrosynthetic 264 network predicted an aldehyde $\mathrm{C}-\mathrm{C}$ ligase reaction between glyoxylate, a common metabolite, 265 and isobutane, a non-metabolite. Inspired by this reaction, an additional simulation was 266 attempted with glyoxylate and prenol, an isoprenoid derivative that contains a C-C double bond 267 to a tertiary carbon that could potentially be ligated to glyoxylate. Though it was hypothesized 268 that this bond would activate $\mathrm{C}-\mathrm{C}$ bond formation with glyoxylate, the prenol-based network 269 converged to a range of pathways to synthesize isobutane from prenol, followed by the same 270 isobutane pathway. Both are shown in Supplementary D.

\section{$271 \quad 3.3$ Tenofovir}


273 reverse transcriptase inhibitor. This molecule is distinct as it contains two chemical moieties 274 uncommon in native metabolism: an organophosphate and an ether bond. The formation of 275 organophosphate bonds was catalyzed by phosphenolpyruvate mutases, producing 276 phosphonopyruvate. ${ }^{38}$ However, the initial set of BNICE operators did not encode for the 277 formation of an ether bond, which prevented pathway resolution.

279 used to successfully find pathways, including a step for the formation of the ether bond (Figure 280 4). Because the broader specificity resulted in many more predicted reactions, multistep 281 simulations became computationally intractable. To counter this, analysis was conducted one 282 generation at a time with manual curation between each step. Because manual curation precludes 283 the use of large pools of substrates for subsequent generational steps, there were fewer 284 redundancies in this pathway than was the case with the prior molecules. Potential enzyme285 substrate pairs were found for each step as shown in Table 3. Steps 1-5 involve phosphorylating 286 the alcohol moieties on 1,2 propanediol, a molecule with demonstrated biosynthetic routes. ${ }^{40}$ 287 One phosphate is used with a transferase to bring in the aforementioned phosphonopyruvate 288 (Step 6), creating the ether bond that was not possible with the original operator set. The other is 289 used to exchange in the adenine nucleotide base (Step 7).

In addition to the production routes for tenofovir, the broadened operator set was also 291 used to develop pathways for the biosynthesis of two tenofovir prodrugs: tenofovir alafenamide 292 fumarate (TAF) and tenofovir disoproxil fumarate (TDF). Both pathways were assembled 293 starting from the tenofovir drug product structure itself, as the final prodrugs do not alter the core 294 of the molecule. For TAF, because the change involves a symmetric addition of two carbonate- 
295 based sidechains, the pathway is relatively short. For TDF, the synthesis is more complex to

296 allow for two distinct attached chains. These paths are presented in Supplementary F.

$297 \quad 3.4$ Yield and Thermodynamics Analysis

Yield calculations varied between the different pathways and, in some cases, variations in

299 yield between pathways for the same molecules were also present. For all possible pathways

300 from the BNICE simulations, a merged overall reaction was used in a flux balance analysis

301 within the iAF1260 model. For most pathways, the starting substrates were already in the model,

302 so yields were calculated based on glucose uptake. In a few cases the model had to be modified

303 to account for metabolites not within the E. coli model but known to exist elsewhere in 304 metabolism. Variation across pathways was a result of analyzing pathways of multiple lengths, 305 and cases where multiple enzyme classes, with different cofactor usage, were predicted to 306 perform the same chemical reaction. Ethambutol showed the lowest yield of 0.15

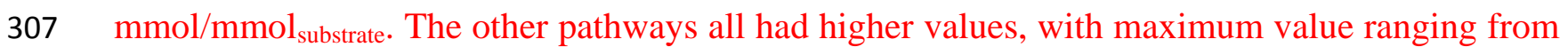
308 0.47-0.85. Interestingly, pyrazinamide showed the highest theoretical maximum yield of 0.85. 309 Overall reactions, and how the yields varied between the individual pathways for each drug 310 product, are available in Supplementary $\mathbf{H}$.

The thermodynamic predictions for all reactions were considered to be relevant and 312 feasible for the directions shown in the figure of this paper. The highest $\Delta \mathrm{G}_{\mathrm{r}} \mathrm{was} 5.22 \mathrm{kcal} / \mathrm{mol}$, 313 which was the result for the reduction of carbonyls to alcohols, EC class 1.1.1. Not only is it 314 common for enzymes in this family to demonstrate reversibility, but this value is also below 315 similar thresholds used by others. ${ }^{16}$ We therefore do not anticipate thermodynamics to be an 316 obstacle for these predicted reactions, which is not surprising since all reaction rules are based on 
317 chemistries known to occur biochemically, and the operators were originally designed with 318 reaction directionality taken into account.

319 4. Discussion

\subsection{Flexible and Rigid Regimes Present Themselves in Multiple Pathways}

Several of the pathway networks partitioned into two regimes, described here as flexible 322 and rigid. In the flexible regime, pathways typically utilize diverse operators/reactions, have 323 reactions that show some order-independence of reactions, and typically diverge from or 324 converge to a singular compound. This can be seen particularly well in the network for tert325 leucine in Figure 5. In part A, the pathway used 5 different chemical changes, illustrated by the 3265 different colored arrows, that tolerated varying substrates throughout the path. The rigid 327 regime, shown in $\mathrm{B}$, involved a much stricter series of reactions, suggesting there were fewer 328 biochemical options available to achieve these transformations and specific ordering was 329 required. The rigid regimes are stricter than the flexible ones in the molecular substructures that 330 are required for the reactions to proceed.

The two regimes were typically evident in the larger pathways that were found. The

332 alternative route presented in Supplementary D for prenol + glyoxylate synthesis explored a 333 wide range of chemical transformations until converging to the same rigid isobutane pathway. 334 Similarly, in an expanded network for the production of pyrazinamide (Supplementary E), the 335 two regimes can also be seen, but reversed; The first two steps (the same as in Figure 2) create 336 (pyrazine-2-yl)methanol, which is further catalyzed more broadly to arrive at the final drug 337 molecule. 
The tendency for these BNICE networks to proceed in flexible vs. rigid regimes can 339 influence future pathway development for similar applications. While the current approach

340 removes intermediates that fall below the averaged similarity of each generational pool ${ }^{18}$, a 341 different methodology could isolate similarity outliers with scores much higher than the rest and 342 artificially enter the rigid regime, allowing the development of longer pathways in a shorter time. 343 Additionally, the operators can now be generated to encode varying levels of molecular sub344 structure specificity in the recognized active sites. ${ }^{41}$ Networks could artificially create this 345 flexible and rigid trend by selective use of the low specificity operators for flexible regimes, and 346 high specificity for rigid.

Flexible and rigid regimes can also inform experimental studies. The rigid regime 348 inherently has fewer enzymatic options, and thus is a reasonable point to begin proof-of-concept 349 studies. If these reactions fail, then there are limited alternative enzyme-substrate options 350 available, and the pathway should be rejected. Alternatively, a reaction that can not be 351 implemented experimentally can be re-run in a BNICE simulation to find multi-step alternative 352 pathways that avoid the unsuccessful reaction. The flexible regime, however, is better for later 353 experiments, as the wide variety of substrate-enzyme pairs will provide alternatives should one 354 reaction fail. Many combinations of enzymes and substrates can be studied in parallel for the 355 flexible regimes, while the rigid steps will be controlled more strongly by biochemical 356 feasibility, independent of enzymatic capabilities.

\section{4.2 Designed Pathways and Natural Pathways Use Enzyme Classes at the Same Frequency}

Although our synthetic targets were all non-natural, the biochemical pathways exhibit 
pathways were representative of the most populous EC classes. The top four operators used in the networks all fell within the top ten largest EC classes (1.1.1, 1.14.13, 2.1.1, and 4.2.1), and are shown with arrows colored red, blue, green, and purple, respectively, in Figures 2-3. Beyond that, the rest of the operators present in the pathways were within the top $9 \%$ of the largest thirdlevel EC families. This suggests that our coverage of enzymatic data is strong for EC families with the broadest substrate representation. The largest classes, with the most available reactions to generalize, gave us the broadest rules. These larger classes (and rulesets) are useful for biosynthesis as they describe chemistries achievable through catalysis on a wide range of substrates. However, these broader enzyme classes may be dominating the network generation, as they generate so many pathway intermediates as to make network generation of long pathways computationally intractable. The remaining less extensive classes contain many interesting potential biochemistries. Smaller EC classes present a challenge, as BNICE operators attempt to generalize observations. Without as many examples, operators may be overly conservative with regard to the substrates they will accept, even if the enzymes are more promiscuous. Only by broadening the experimental observations of an EC class, or by loosening the generalization criteria beyond the EC system for these smaller enzyme families, can we enhance the utilization of particular biochemistries.

\subsection{Challenges in Assembling Large Molecules}

Successful pathways rarely contain large changes in molecular size or weight. This is partially a result of the focus on chemically similar starting compounds; however, even in the cases where networks did have a large change in molecular weight, like the $\mathrm{N}$-alkyl transferase in the ethambutol pathway (Supplementary G), enzymatic options for those biosynthetic steps were restrictive. Networks tended to converge on those points, suggesting that biosynthesis, or 
383 our current rule set at the very least, contains many rules that excel at chemical modifications 384 and changes in functional groups but are lacking in anabolic biochemistry (i.e., $\mathrm{C}$-C bond 385 forming). This is reflective of native biochemistry as well, as four out of the top five largest

native EC families are involved in either the modification or transfer of small functional groups: alcohol oxidoreductions (1.1.1), methylation (2.1.1), acetyl-CoA mediated acetylation (2.3.1), and hydroxylation (1.14.13). These four classes, representing roughly $20 \%$ of the defined reactions in KEGG, either do not alter carbon numbers, or do so by no more than two.

Implementing the designed pathways into experimental systems could provide exciting new opportunities for pharmaceutical synthesis, though a major challenge will be ensuring enzyme promiscuity towards non-native substrates. Here, we use SimZyme to identify enzymes with substrates that are chemically similar to the non-native pathway substrates. Our own internal experimental studies have shown roughly a success rate of $75 \%$ for these predictions (data not shown). A range of other strategies can be used to find particularly promiscuous enzymes, such as: utilizing known promiscuous superfamilies, ${ }^{42}$ predicting based on amino acid sequence, $^{43}$ identifying ancestrally divergent enzymes ${ }^{44}$, or engineering broader substrate specificity. ${ }^{45}$ The kinetics of the promiscuous rates are likely much lower than would be practical for industrial systems. Fortunately, these types of reactions are prime targets for rational engineering or directed evolution to increase the desired activity. ${ }^{46}$

Computational simulations have had many successful applications for informing novel metabolic engineering strategies. Computational strategies for the engineering of metabolic encompass a wide range of approaches. Some have focused on novel strain design strategies, ${ }^{47}$ pathway control, ${ }^{48}$ and even applying kinetic simulations. ${ }^{49}$ While all of these approaches and others $^{9}$ are very useful and important, most pathway design methodologies do not allow for 

with respect to strain design and engineering. However, to the author's knowledge, no other

414 publications have developed pathways for molecules as heterologous to metabolism as those in 415 this study, evidenced by the significantly longer pathways necessary for connecting these 416 molecules to known metabolites. While this does increase the experimental difficulties in 417 implementing such pathways, this work shows that the reaches of putative biochemistry are 418 farther than have been shown thus far.

\section{$419 \quad \underline{5 . \text { Conclusions }}$} substrates that were required. Further, the set of operators that were represented in our

427 simulations aligned with the broadest types of chemistry known to occur natively. Nearly all 
428 predictions were connected to EC classes containing chemically similar substrates with 429 confirmed activity.

Experimental validation of these types of pathways will, of course, be necessary.

431 Implementing these pathways will require the ability to identify, express, purify, and chemically

432 characterize reaction products from a large number of potential enzymes. High-throughput

433 technologies make this increasingly feasible, but a particularly difficult obstacle will be the

434 necessity to synthesize chemical pathway intermediates to allow for the testing of multiple

435 reactions concurrently. Successes will necessitate the cooperation of not only biochemists, but

436 also organic chemists. Custom synthesis resources will also be useful, though the ability to

437 synthesize some molecules will likely still be intractable.

The BNICE platform is an extensive, robust, and powerful tool for the development of

439 these types of pathways. Further developments in the operators, as well as developments in the

440 ability to accurately predict promiscuous enzyme activities, will enable the implementation of

441 these and similarly developed pathways to change how chemicals are made. Limitations in the

442 design of biochemical pathways that arise from inaccessible chemistry can serve as guideposts

443 for future work in substrate selection. Though it is difficult to definitively state the absolute

444 limits of biochemistry, difficulties with engineering rarer reactions, like promiscuous $\mathrm{C}-\mathrm{C}$ bond

445 formation, can inform future pathway development and drive the selection of starting substrates,

446 selective operator sets, or the future engineering of new enzymatic reactions.

447 6. Acknowledgements

The authors would like to thank the Center for Global Health at Northwestern;

449 specifically Dr. Robert Murphy and Dr. Kimberly Scarsi, and the TB Alliance, specifically Dr. 
450 Christopher Cooper and Dr. Takushi Kaneko at the TB Alliance for helpful conversations on 451 selecting priority drugs, and Andrew Stine for the use of his automated operator sets. Funding 452 was provided by the Bill and Melinda Gates Foundation, NSF CBET-0835800, and the 453 Northwestern McCormick School of Engineering.

$454 \quad$ 7. List of Tables

455 Table 1: Matched substrates for predicted biosynthesis paths for pyrazinamide. When multiple 456 operators performed the same reaction, the SimZyme matched EC number is bold with 457 alternatives in parentheses.

458 Table 2: Matched substrates for predicted biosynthesis paths for L-tert leucine. When multiple 459 operators performed the same reaction, the SimZyme matched EC number is bold with 460 alternatives in parentheses. Reaction 17 was the single case of an unsuccessfully matched 461 substrate, with the closest match shown with the $\pi$-bond of the carbonyl overlaying the $\pi$-system 462 of the aromatic ring. $\mathrm{R}_{1}$ is a carotenoid-based tail.

463 Table 3: Matched substrates for predicted biosynthesis paths for tenofovir. Reaction 5 contains 464 only three EC digits because the studied enzyme has only been assigned those numbers. ${ }^{52}$

\section{8. List of Figures}

466 Figure 1: Diagram of the work flow for pathway development with BNICE and SimZyme. (A) 467 High-impact targets were selected based on information from public health resources, (B) 468 BNICE was then used to find pathways to those targets, and (C) SimZyme then used chemical 469 similarity to known, active substrates to suggest isozyme candidates. 
470 Figure 2: Predicted paths for the biosynthesis of pyrazinamide. Arrows are colored to show the

471 top four EC classes within all pathways generated. Red - 1.1.1, Blue - 1.14.13, Green - 2.1.1

472 Figure 3: Predicted paths for the biosynthesis of L-tert leucine. Arrows are colored to show the

473 top four EC classes within all pathways generated. Red - 1.1.1, Blue - 1.14.13, Green - 2.1.1,

$474 \quad$ Purple -4.2 .1$

475 Figure 4: Predicted paths for the biosynthesis of tenofovir.

476 Figure 5: An illustration of the "flexible" (A) and "rigid" (B) regimes within the tert-leucine

477 pathway. Within the flexible regime, colored arrows correspond to the same chemistry occurring

478 on multiple substrates. The orange dashed line represents a multi-step substitute for the oxidation

479 of an alcohol to an aldehyde, through a methoxy intermediate.

480

481

9. References

482 1. Ajikumar, P. K. et al. Isoprenoid pathway optimization for taxol precursor overproduction 483 in Escherichia coli. Science 330, 70-4 (2010).

484

485

486

2. Ro, D.-K. et al. Production of the antimalarial drug precursor artemisinic acid in engineered yeast. Nature 440, 940-3 (2006).

487

488

3. Patel, R. N., Chu, L. \& Mueller, R. Diastereoselective microbial reduction of (S)-[3-

489

490 chloro-2-oxo-1-(phenylmethyl)propyl]carbamic acid, 1,1-dimethylethyl ester.

Tetrahedron: Asymmetry 14, 3105-3109 (2003).

491

492 4. Zhang, K., Li, H., Cho, K. M. \& Liao, J. C. Expanding metabolism for total biosynthesis

493 of the nonnatural amino acid L-homoalanine. Proc. Natl. Acad. Sci. U. S. A. 107, 6234-9

494 (2010). 
496 5. Jin, J. Z. \& Zhang, J. Highly enantioselective hydrolysis of racemic isopropyl tertleucinate by newly discovered Baclicus lincheniformis Jx010 for synthesis of L-tert-butyl leucine. Adv. Mater. Res. 343-344, 453-456 (2011).

499

500

6. Patel, R. N. Biocatalysis: Synthesis of key intermediates for development of pharmaceuticals. ACS Catal. 1, 1056-1074 (2011).

502

503

504

505

506

507

508

509

510

511

512

513

514

515

516

517

518
7. Hult, K. \& Berglund, P. Enzyme promiscuity: mechanism and applications. Trends Biotechnol. 25, 231-8 (2007).

8. Nam, H. et al. Network context and selection in the evolution to enzyme specificity. Science 337, 1101-4 (2012).

9. Moura, M., Broadbelt, L. \& Tyo, K. in Methods in Molecular Biology - Systems Metabolic Engineering: Methods and Protocols (ed. Alper, H. S.) 985, 123-147 (2013).

10. Medema, M. H., van Raaphorst, R., Takano, E. \& Breitling, R. Computational tools for the synthetic design of biochemical pathways. Nat. Rev. Microbiol. 10, 191-202 (2012).

11. Hadadi, N. \& Hatzimanikatis, V. Design of computational retrobiosynthesis tools for the design of de novo synthetic pathways. Curr. Opin. Chem. Biol. 28, 99-104 (2015).

12. Keasling, J. D. Synthetic biology and the development of tools for metabolic engineering. Metab. Eng. 14, 189-195 (2012).

13. Carbonell, P., Parutto, P., Herisson, J., Pandit, S. B. \& Faulon, J.-L. XTMS: pathway design in an eXTended metabolic space. Nucleic Acids Res. 42, W389-94 (2014).

14. Jeffryes, J. G. et al. MINEs: Open access databases of computationally predicted enzyme 
promiscuity products for untargeted metabolomics. J. Cheminform. in press, (2015).

526

527

528

529

530

531

532

533

534

535

536

537

538

539

540

541

542

543

544

545

546

547

548

549

550

551

552

553

554

15. Moriya, Y. et al. PathPred: an enzyme-catalyzed metabolic pathway prediction server. Nucleic Acids Res. 38, W138-43 (2010).

16. Campodonico, M. A., Andrews, B. A., Asenjo, J. A., Palsson, B. O. \& Feist, A. M. Generation of an atlas for commodity chemical production in Escherichia coli and a novel pathway prediction algorithm, GEM-Path. Metab. Eng. 25, 140-158 (2014).

17. Hatzimanikatis, V. et al. Exploring the diversity of complex metabolic networks. Bioinformatics 21, 1603-9 (2005).

18. Pertusi, D. A., Stine, A. E., Broadbelt, L. J. \& Tyo, K. E. J. Efficient searching and annotation of metabolic networks using chemical similarity. Bioinformatics 31, 10161024 (2014).

19. Henry, C. S., Broadbelt, L. J. \& Hatzimanikatis, V. Discovery and analysis of novel metabolic pathways for the biosynthesis of industrial chemicals: 3-hydroxypropanoate. Biotechnol. Bioeng. 106, 462-73 (2010).

20. Wu, D., Wang, Q., Assary, R. S., Broadbelt, L. J. \& Krilov, G. A computational approach to design and evaluate enzymatic reaction pathways: application to 1-butanol production from pyruvate. J. Chem. Inf. Model. 51, 1634-47 (2011).

21. Finley, S. D., Broadbelt, L. J. \& Hatzimanikatis, V. In silico feasibility of novel biodegradation pathways for 1,2,4-trichlorobenzene. BMC Syst. Biol. 4, 7 (2010).

22. World Health Organization. Global Price Reporting Mechanism. (World Health Organization, 2010). at <http://www.who.int/hiv/amds/gprm/en/> 
555 23. Jankowski, M. D., Henry, C. S., Broadbelt, L. J. \& Hatzimanikatis, V. Group contribution

556

557

558

559

560

561

562

563

564

565

566

567

568

569

570

571

572

573

574

575

576

577

578

579

580

581

582

583

584 method for thermodynamic analysis of complex metabolic networks. Biophys. J. 95, 1487-99 (2008).

24. Hattori, M., Tanaka, N., Kanehisa, M. \& Goto, S. SIMCOMP/SUBCOMP: chemical structure search servers for network analyses. Nucleic Acids Res. 38, W652-6 (2010).

25. Feist, A. M. et al. A genome-scale metabolic reconstruction for Escherichia coli K-12 MG1655 that accounts for 1260 ORFs and thermodynamic information. Mol. Syst. Biol. 3, 121 (2007).

26. Kanehisa, M. \& Goto, S. KEGG: Kyoto encyclopedia of genes and genomes. Nucleic Acids Res. 28, 27-30 (2000).

27. Byers, T. \& Waterman, M. Technical Note-Determining All Optimal and Near-Optimal Solutions when Solving Shortest Path Problems by Dynamic Programming. Oper. Res. 32, 1381-1384 (1984).

28. Stine, A. \& Broadbelt, L. J. personal communication.

29. Scheer, M. et al. BRENDA, the enzyme information system in 2011. Nucleic Acids Res. 39, D670-6 (2011).

30. O’Boyle, N. M. et al. Open Babel: An open chemical toolbox. J. Cheminform. 3, 33 (2011).

31. Willett, P. Similarity-based virtual screening using 2D fingerprints. Drug Discov. Today 11, 1046-53 (2006).

32. Vlessis, A. A., Bartos, D. \& Trunkey, D. Importance of spontaneous $\alpha$-ketoacid 
607

608

609

610

611

612

613

614

decarboxylation in experiments involving peroxide. Biochem. Biophys. Res. Commun. 170, 1281-1287 (1990).

33. Tucker, J. S. \& Morgan, M. E. Decarboxylation of alpha-keto acids by Streptococcus lactis var. maltigenes. Appl. Microbiol. 15, 694-700 (1967).

34. Schellenberger, J. et al. Quantitative prediction of cellular metabolism with constraintbased models: the COBRA Toolbox v2.0. Nat. Protoc. 6, 1290-307 (2011).

35. Dickschat, J. S. et al. Pyrazine biosynthesis in Corynebacterium glutamicum. European J. Org. Chem. 2010, 2687-2695 (2010).

36. Martín, M. J. et al. Isolation and first total synthesis of PM050489 and PM060184, two new marine anticancer compounds. J. Am. Chem. Soc. 135, 10164-10171 (2013).

37. Njoroge, F. G., Chen, K. X., Shih, N.-Y. \& Piwinski, J. J. Challenges in modern drug discovery: a case study of boceprevir, an HCV protease inhibitor for the treatment of hepatitis C virus infection. Acc. Chem. Res. 41, 50-9 (2008).

38. Liu, S., Lu, Z., Jia, Y., Dunaway-Mariano, D. \& Herzberg, O. Dissociative phosphoryl transfer in PEP mutase catalysis: Structure of the enzyme/sulfopyruvate complex and kinetic properties of mutants. Biochemistry 41, 10270-10276 (2002).

39. Caspi, R. et al. The MetaCyc database of metabolic pathways and enzymes and the BioCyc collection of pathway/genome databases. Nucleic Acids Res. 42, D459-71 (2014).

40. Altaras, N. E. \& Cameron, D. C. Metabolic engineering of a 1,2-propanediol pathway in Escherichia coli. Appl. Envir. Microbiol. 65, 1180-1185 (1999).

14 41. Stine, A. E. Ph.D. Dissertation. (Northwestern University, 2015). 
616 42. Jonas, S. \& Hollfelder, F. Mapping catalytic promiscuity in the alkaline phosphatase superfamily. Pure Appl. Chem. 81, 731-742 (2009).

618

619

43. Carbonell, P. \& Faulon, J.-L. Molecular signatures-based prediction of enzyme

620 promiscuity. Bioinformatics 26, 2012-9 (2010).

621

622 44. Khersonsky, O. \& Tawfik, D. S. Enzyme promiscuity: a mechanistic and evolutionary perspective. Annu. Rev. Biochem. 79, 471-505 (2010).

624

625

626

45. Hunter, D. J. B. et al. Facile production of minor metabolites for drug development using a CYP3A shuffled library. Metab. Eng. 13, 682-93 (2011).

627

628

46. Lippow, S. M. et al. Engineering Enzyme Specificity Using Computational Design of a Defined-Sequence Library. Chem. Biol. 17, 1306-1315 (2010).

630

631

632

633

634

635

636

637

638

639

640

641

642

643

644
47. Yang, L., Srinivasan, S., Mahadevan, R. \& Cluett, W. R. Characterizing metabolic pathway diversification in the context of perturbation size. Metab. Eng. 28, 114-22 (2015).

48. Watstein, D. M., McNerney, M. P. \& Styczynski, M. P. Precise metabolic engineering of carotenoid biosynthesis in Escherichia coli towards a low-cost biosensor. Metab. Eng. 31, 171-180 (2015).

49. Nikolaev, E. V. The elucidation of metabolic pathways and their improvements using stable optimization of large-scale kinetic models of cellular systems. Metab. Eng. 12, 2638 (2010).

50. Yim, H. et al. Metabolic engineering of Escherichia coli for direct production of 1,4butanediol. Nat. Chem. Biol. 7, 445-452 (2011). 
646 51. Cho, A., Yun, H., Park, J. H., Lee, S. Y. \& Park, S. Prediction of novel synthetic pathways 647 for the production of desired chemicals. BMC Syst. Biol. 4, 35 (2010).

648

649 52. Lehmacher, A. \& Hensel, R. Cloning, sequencing and expression of the gene encoding 2650 phosphoglycerate kinase from Methanothermus fervidus. MGG Mol. Gen. Genet. 242, $651 \quad 163-168$ (1994).

652 
Target Identification

A)

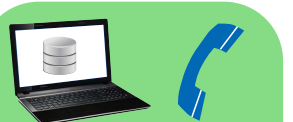

Gates Foundation TB Alliance Northwestern WHO
Pathway Identification

B)

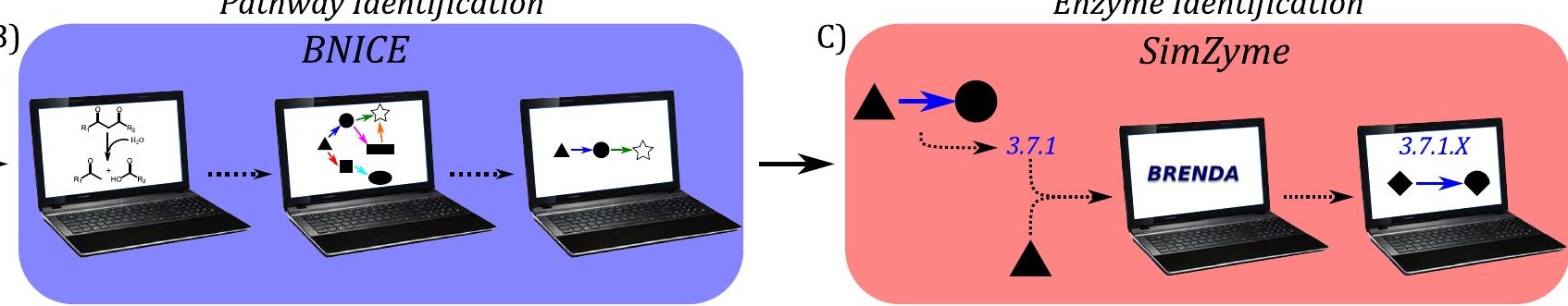


Figure2

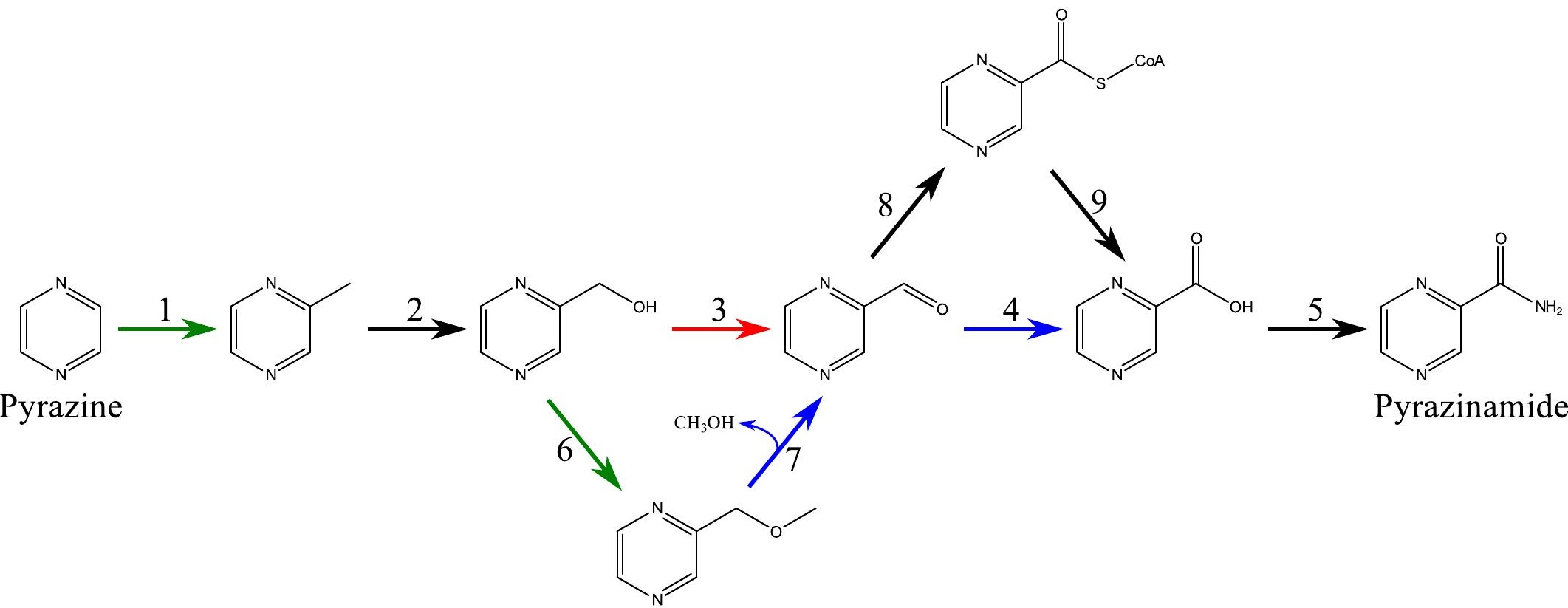




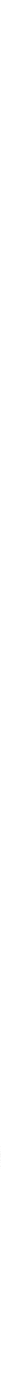




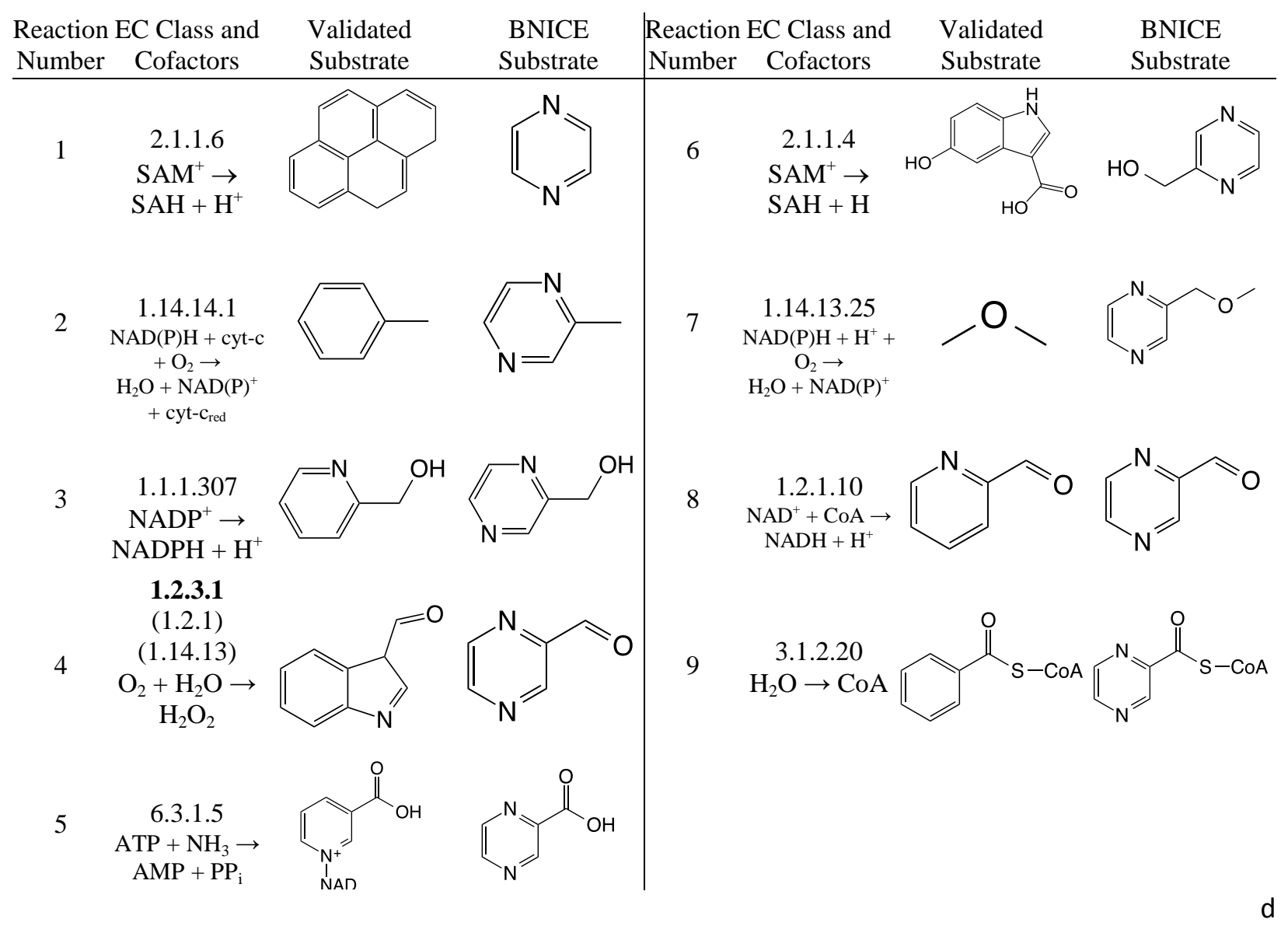




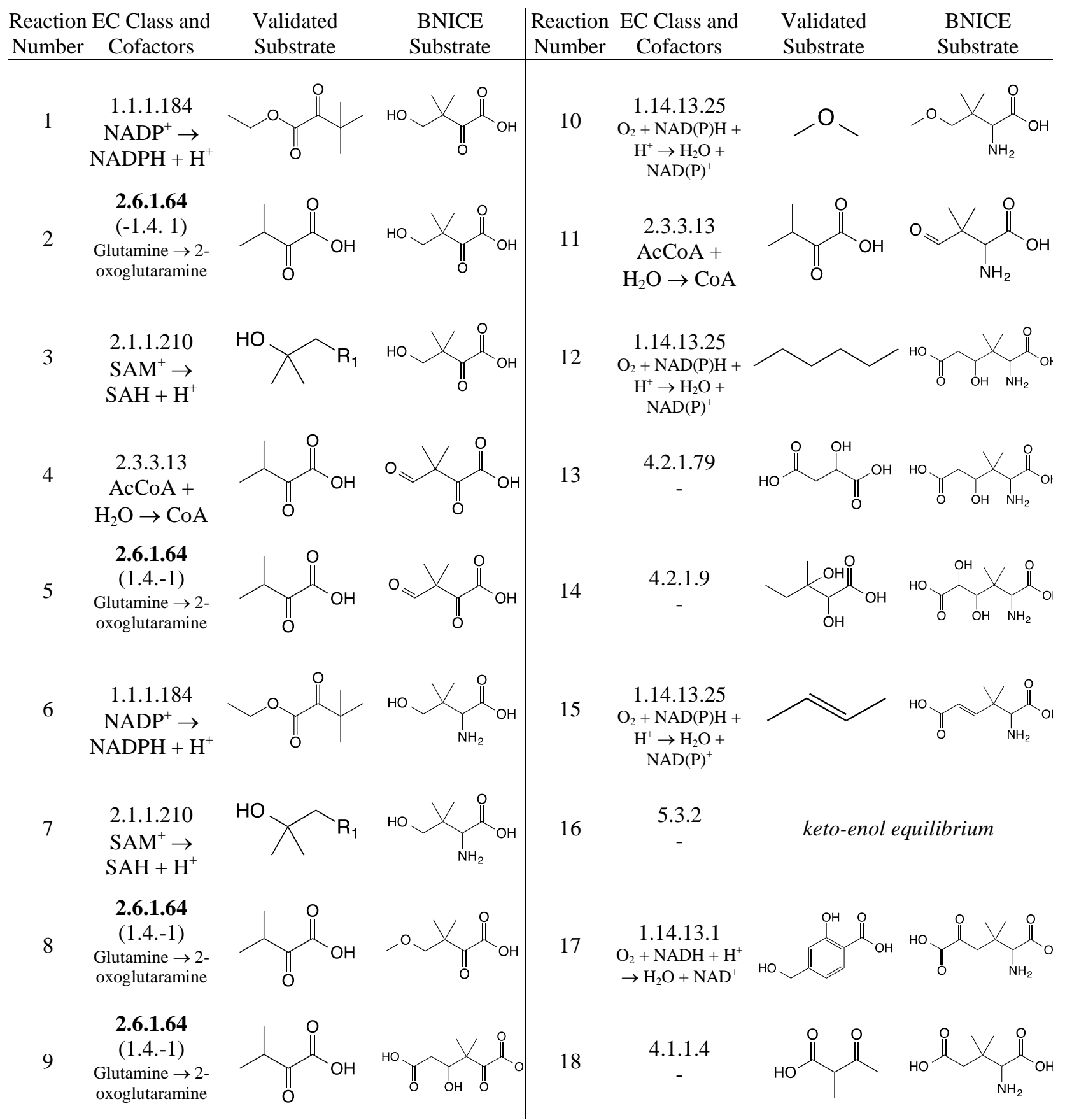




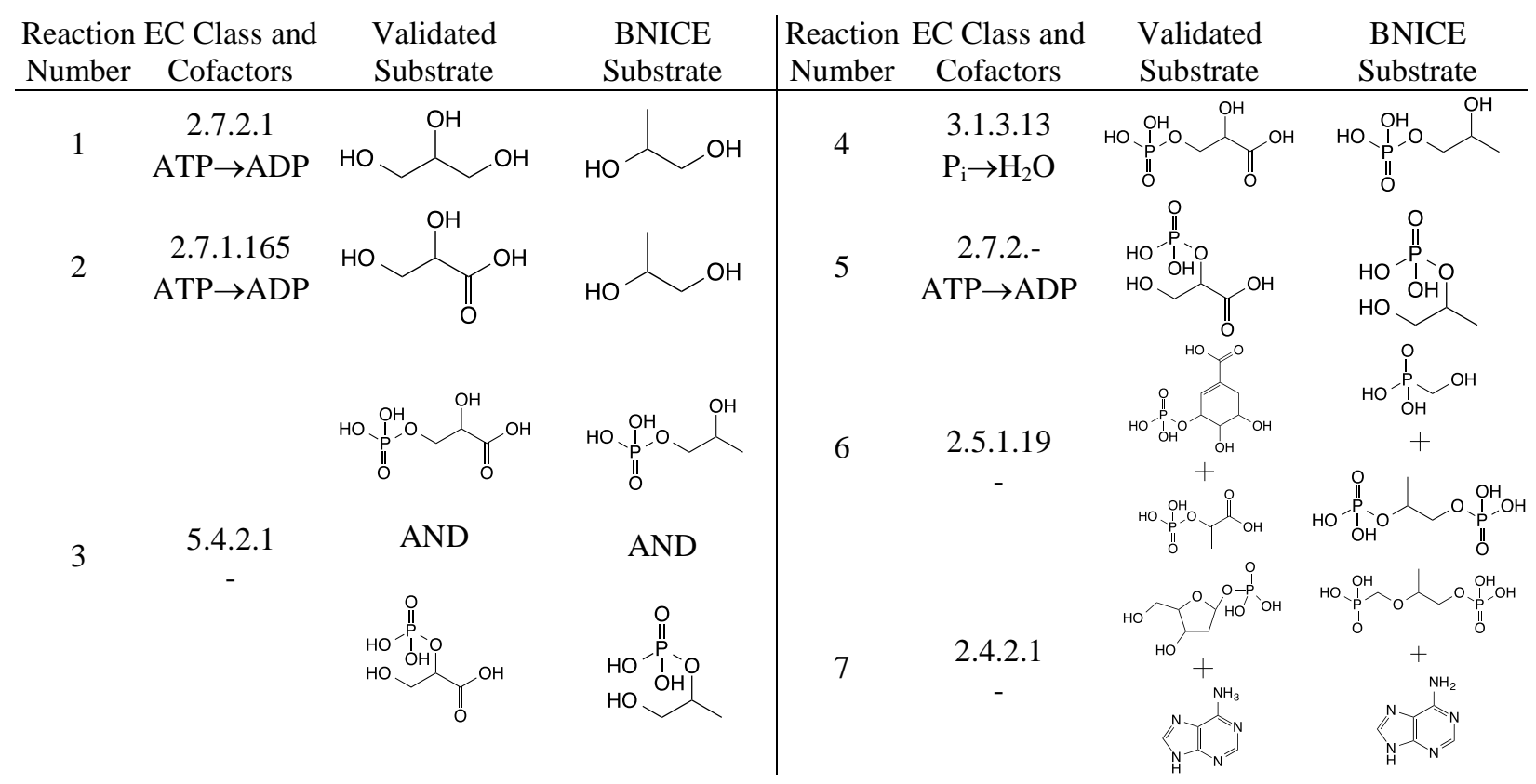




\begin{tabular}{ccc}
\hline Drug & $\begin{array}{c}\text { Max Yield Range } \\
\left(\mathbf{m m o l}_{\text {prod }} \mathbf{m m o l}_{\text {C-source }}\right)\end{array}$ & Carbon Source \\
\hline Ethambutol & 0.15 & 2,3 -diaminopropanoate $^{*}$ \\
Pyrazinamide & $0.37-0.85$ & Pyrazine $^{* *}$ \\
Didanosine & $0.3-0.52$ & Glucose \\
Stavudine & $0.54-0.55$ & Glucose \\
Tert-leucine & $0.35-0.63$ & Glucose \\
Tenofovir & 0.47 & Glucose
\end{tabular}

